Bundesgesundheitsbl 2020 $63: 1203-1218$ https://doi.org/10.1007/s00103-020-03215-7 Eingegangen: 10. März 2020

Angenommen: 24. August 2020

Online publiziert: 22. September 2020

(c) Der/die Autor(en) 2020

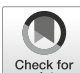

\title{
Alfons Hollederer
}

Fachbereich 01 Humanwissenschaften, Professur Theorie und Empirie des Gesundheitswesens, Universität Kassel, Kassel, Deutschland

\section{Die Gewährleistung von Krankheitshilfen bei asylsuchenden Menschen: Zweiklassenmedizin in Deutschland?}

für wirtschaftliche, soziale und kulturelle Rechte der Vereinten Nationen drückt seine Besorgnis aus, dass „asylum-seekers' access to health care is restricted to acute and painful conditions for the first 15 months of their stay in Germany and that their access to health care is further limited ..." [9].

Die Versorgung von Flüchtlingen ist aufgrund der föderalen Struktur in Deutschland durch verschiedene $\mathrm{Zu}$ ständigkeiten auf der Bundes-, Länderund kommunalen Ebene geprägt. Für die Durchführung des Asylverfahrens ist der Bund bzw. das Bundesamt für Migration und Flüchtlinge (BAMF) zuständig. Die Verteilung der ankommenden Flüchtlinge auf die Bundesländer orientiert sich an dem Königsteiner Schlüssel und damit nach Steueraufkommen und Bevölkerungszahlen. Die Länder sind nach dem Asylverfahrensgesetz verpflichtet, für die Unterbringung von Asylsuchenden $\mathrm{zu}$ sorgen und die Geld- und Sachleistungen $\mathrm{zu}$ ihrer Existenzsicherung zu gewährleisten. Nach der Entlassung aus der Aufnahmeeinrichtung folgt die Verteilung innerhalb des Landes in Gemeinschaftsunterkünfte und dezentrale Unterbringungsformen. Es bestehen erhebliche Unterschiede in der Administration zwischen den Bundesländern und in den Kommunen. Asylbewerber und sonstige ausländische Flüchtlinge, die keinen dauerhaften Aufenthaltsstatus besitzen, erhalten im Falle der Hilfsbe- dürftigkeit soziale Leistungen nach Maßgabe des vorrangigen Asylbewerberleistungsgesetzes (AsylbLG). Der Kreis der Leistungsberechtigten, die sich tatsächlich im Bundesgebiet aufhalten müssen, ist nach $\$ 1$ Abs. 1 AsylbLG normiert und mit Voraussetzungen verbunden. Leistungsberechtigt sind Ausländer, die ein Asylgesuch geäußert haben bzw. eine Aufenthaltsgestattung nach dem Asylgesetz, eine Aufenthaltserlaubnis oder eine Duldung nach dem Aufenthaltsgesetz besitzen. $\mathrm{Zu}$ den leistungsberechtigten Gruppen zählen auch Personen, die über einen Flughafen einreisen wollen und denen die Einreise noch nicht gestattet ist. Weiterhin sind Personen leistungsberechtigt, die vollziehbar ausreisepflichtig sind oder einen Folgeantrag auf Asyl stellen. Hinzu kommen die Ehegatten, Lebenspartner oder minderjährigen Kinder der leistungsberechtigten Personen, ohne dass sie selbst die Voraussetzungen erfüllen.

Das Zugangsverfahren zu medizinischen Leistungen ist in den Bundesländern verschieden reguliert. Für den Arztbesuch benötigen die Leistungsberechtigungen meist einen Berechtigungsoder Behandlungsschein, dessen Voraussetzungen zur Ausstellung die zuständige Behörde selbst prüft und entscheidet. Darüber hinaus ist nicht nur der Zugang zur Gesundheitsversorgung, sondern auch die freie Arztwahl eingeschränkt. Außerdem dürfen die Allgemeinmedi- 
ziner im Krankenscheinverfahren in der Regel nicht zu Fachärzten überweisen. Der Übergang von einzelfallbezogenen Krankenscheinausgaben auf ein modernes Gesundheitskartensystem ist allerdings in einem Großteil der Bundesländer in Kooperation mit der gesetzlichen Krankenversicherung gelungen (Landesrahmenvereinbarungen nach $\$ 264$ Abs. 1 Sozialgesetzbuch (SGB) V aktuell in Berlin, Brandenburg, Bremen, Hamburg, Niedersachsen, NordrheinWestfalen, Rheinland-Pfalz, SchleswigHolstein und Thüringen). In der Umsetzung der gesetzlichen Vorgaben des AsylbLG werden im Verwaltungsvollzug die Ermessensspielräume unterschiedlich genutzt. Razum et al. [10] drücken es pointiert so aus, dass der Zufall über den Zugang zur Gesundheitsversorgung für Flüchtlinge in Abhängigkeit von den Zuweisungen des Bundeslandes und der Kommune sowie der juristischen Kenntnisse der behandelnden Ärzte entscheidet. Eine Befragung von Gesundheitsämtern in Deutschland ergab, dass die gesundheitliche Versorgung von Asylsuchenden in Deutschland auBerordentliches Verbesserungspotenzial aufweist [6]. So fehlten strukturelle Ressourcen für die Koordination der Versorgung in den zuständigen Ämtern. Als verbesserungsbedürftig wurden die Standardisierung in den Abläufen, zügige Durchführung von Impfungen, standardisierte Erhebung und Übermittlung gesundheitsbezogener Informationen sowie Konzentration auf wichtige Infektionskrankheiten erachtet [6]. Es hat sich auch herausgestellt, dass die auslösenden Infektionskrankheiten bei Ausbrüchen in Gemeinschaftsunterkünften für Asylsuchende häufig erst in Deutschland erworben wurden [11].

In Deutschland liegen zur Gesundheitsversorgung von Asylsuchenden und Flüchtlingen nur wenige repräsentative Daten und systematische Gesundheitsberichte vor [7]. Empirischen Arbeiten zur medizinischen Versorgung von Asylsuchenden kommt daher besondere Wichtigkeit zu [12]. Die Sekundärdatenanalyse zielt deshalb darauf, das Leistungsgeschehen im Krankheitsfall bei den Leistungsempfängern nach dem AsylbLG zu explorieren sowie nach regionalen, sozialen und gesundheitssystemischen Unterschiedshypothesen $\mathrm{zu}$ analysieren. Die Gewährung von Krankheitshilfen bei asylsuchenden Menschen ist immanent mit den Asylgesetzen und der Sozialgesetzgebung verbunden, die nicht nur das Leistungsrecht, sondern auch die Begriffe und die Bundesstatistiken determinieren. Die vorliegende Arbeit führt die Daten separater Bundesstatistiken zusammen und hat die Analyse verschiedener Gewährleistungsprinzipien in der Gesundheitsversorgung nach Reichweite, Regionen, Bruttoausgaben und Gesundheitskarte zum Ziel. Es werden erstmalig Prävalenzraten der ambulanten und stationären Hilfen bei Krankheit nach dem AsylbLG für Bundesländer und soziodemografische Gruppen berechnet. Im Abschnitt Methode wird zunächst auf die essenziellen sozialrechtlichen Grundlagen und danach auf die Asylbewerberleistungsstatistiken eingegangen. Es schließen sich im Ergebnisteil die Sekundäranalysen zum Leistungsgeschehen im Krankheitsfall bei den Leistungsempfängern nach dem AsylbLG auf Basis der Forschungsdatensätze an. Die Diskussion der empirischen Ergebnisse und ein Fazit runden die Arbeit ab.

\section{Methode}

Die Asylbewerberregelleistungen werden entweder als a) sogenannte Grundleistungen oder b) in besonderen Fällen in Form von laufender Hilfe zum Lebensunterhalt gewährt:

$\mathrm{Zu}$ a) Die Grundleistungen nach $\$ 3$ AsylbLG werden zur Deckung des notwendigen Bedarfs an Ernährung, Unterkunft, Heizung, Kleidung, Gesundheitspflege sowie Gebrauchs- und Verbrauchsgütern des Haushalts gewährt. Sie werden vorrangig als Sachleistung erbracht. $\mathrm{Zu}$ den Grundleistungen können in einer speziellen Bedarfssituation besondere Leistungen bei Krankheit, Schwangerschaft und Geburt ( $\$ 4$ AsylbLG) und sonstige Leistungen ( $\$ 6$ AsylbLG) genehmigt werden (Stichwort „Minimalversorgung“).

$\mathrm{Zu}$ b) In den besonderen Fällen wird nach $\$ 2$ AsylbLG das Zwölfte Sozialgesetzbuch (SGB XII) auf diejenigen
Leistungsberechtigten angewandt, die sich seit 18 Monaten ohne wesentliche Unterbrechung im Bundesgebiet aufhalten und die Dauer des Aufenthalts nicht rechtsmissbräuchlich selbst beeinflusst haben (die vorgeschriebene Voraufenthaltszeit lag in 2018 noch bei 15 Monaten). $\mathrm{Zu}$ den Analogleistungen gehören Hilfe bei Krankheit, Hilfe zur Pflege, Hilfe bei Schwangerschaft und Mutterschaft. Sie entsprechen weitgehend der Regelversorgung in der gesetzlichen Krankenversicherung (Stichwort „Regelversorgung“).

Die Leistungsberechtigten nach den $\$ \S 3-6$ AsylbLG haben im Vergleich zum SGB XII einen deutlich eingeschränkten Anspruch auf Gesundheitsversorgung, der sich auf die nicht aufschiebbare Akut- und Notfallversorgung konzentriert. Nach $\$ 4$ Absatz 1 AsylbLG sind

zur Behandlung akuter Erkrankungen und Schmerzzustände die erforderliche ärztliche und zahnärztliche Behandlung einschließlich der Versorgung mit Arzneiund Verbandmitteln sowie sonstiger zur Genesung, zur Besserung oder zur Linderung von Krankheiten oder Krankheitsfolgen erforderlichen Leistungen zu gewähren. Zur Verhütung und Früherkennung von Krankheiten werden Schutzimpfungen entsprechend den $\$ \$ 47,52$ Absatz 1 Satz 1 des Zwölften Buches Sozialgesetzbuch und die medizinisch gebotenen Vorsorgeuntersuchungen erbracht. Eine Versorgung mit Zahnersatz erfolgt nur, soweit dies im Einzelfall aus medizinischen Gründen unaufschiebbar ist.

Für werdende Mütter und Wöchnerinnen sind ärztliche und pflegerische Hilfe und Betreuung, Hebammenhilfe, Arznei-, Verband- und Heilmittel zu gewähren ( $\$ 4$ Abs. 2 AsylbLG). Das entspricht der normalen Grundversorgung. Daneben können nach $\$ 6$ Abs. 1 AsylbLG sonstige Leistungen gewährt werden, wenn sie im Einzelfall zur Sicherung der Gesundheit unerlässlich ist. Personen mit einer Aufenthaltserlaubnis und besonderen Bedürfnissen,

wie beispielsweise unbegleitete Minderjährige oder Personen, die Folter, Vergewaltigung oder sonstige schwere Formen 
Bundesgesundheitsbl 2020 -63:1203-1218 https://doi.org/10.1007/s00103-020-03215-7

(c) Der/die Autor(en) 2020

\section{A. Hollederer}

\section{Die Gewährleistung von Krankheitshilfen bei asylsuchenden Menschen: Zweiklassenmedizin in Deutschland?}

\section{Zusammenfassung}

Hintergrund. Es gibt im Bereich des Asylbewerberleistungsgesetzes (AsylbLG) zwei Möglichkeiten der Leistungsgewährung mit praktischer Relevanz für die Gesundheitsversorgung (abhängig von der Voraufenthaltszeit): die Grundleistungen und die Leistungen in besonderen Fällen analog zum Sozialgesetzbuch (SGB) XII.

Methodik. Die Sekundärdatenanalyse untersucht das Leistungsgeschehen im Krankheitsfall bei den Leistungsempfängern nach dem AsylbLG beim Forschungsdatenzentrum der Statistischen Ämter des Bundes und der Länder. Dem untersuchten Personenkreis wurde noch keine Flüchtlingseigenschaft bzw. Asylberechtigung zuerkannt.

Ergebnisse. Zum Stichtag 31.12.2018 bezogen 423.201 Personen in Deutschland Leistungen nach dem AsylbLG. Davon war gut ein Drittel Frauen. Das Durchschnittsalter betrug 24 Jahre. Über die Hälfte stammte aus Asien. Über ein Drittel aller Leistungsempfänger befand sich in ambulanter $(33,5 \%)$ oder stationärer Behandlung (1,3\%). Zwischen den Bundesländern variierten die Leistungen zur Hilfe bei Krankheit sowie die gesundheitsbezogenen Pro-Kopf-Bruttoausgaben sehr stark. Die Gewährung von Leistungen bei Krankheit war in Aufnahmeeinrichtungen relativ gering. Mit Gesundheitskarte war die Inanspruchnahme stationärer Behandlung generell höher.

Die gesundheitsbezogene Regelversorgung mit Hilfen in besonderen Fällen ( $\$ 2$ AsylbLG analog SGB XII) erreichte einen größeren Anteil an leistungsberechtigten Menschen mit 42,7\% am Jahresende als die Minimalversorgung nach $\S 3$ AsylbLG mit $29,0 \%$. Sie verursachte trotzdem im Vergleich weniger Bruttoausgaben.

Schlussfolgerung. Es wird empfohlen, §2 AsylbLG schon bei einer Voraufenthaltszeit ab 3 Monaten anzuwenden, um frühzeitiger die Hilfen analog Kap. 5-9 SGB XII gewähren zu können. Eine flächendeckende Einführung der Gesundheitskarte würde den Zugang verbessern.

Schlüsselwörter

Asylsuchende · Geflüchtete · Zugang zur Gesundheitsversorgung $\cdot$ Menschenrechte

\section{Guarantee of illness assistance for people seeking asylum: Two-tiered medical system in Germany?}

\section{Abstract}

Purpose. In the area of the Asylum Seeker Benefits Act (AsylbLG), there are two options for granting benefits that have practical relevance to health care (depending on the period of previous residence): minimum provision and benefits in special cases, analogous to the Social Code Book XII (SGB XII).

Method. This secondary data analysis examines the performance of benefits in the case of illness among benefit recipients according to the AsylbLG at the Research Data Centre of the Federal and State Statistical Offices. The examined group of individuals have not yet been granted refugee status or asylum entitlement.
Results. As of 31 December 2018, 423,201 persons in Germany were receiving benefits according to the AsylbLG. A good third of these were women. The average age was 24 years, and more than half came from Asia. More than one-third of all benefit recipients were in outpatient $(33.5 \%)$ or inpatient treatment (1.3\%). Among the federal states, benefits for assistance in the case of illness as well as the health-related per capita gross expenditure varied considerably. The provision of illness benefits in reception facilities was relatively low. With the health card, the use of inpatient treatment was higher. The standard healthrelated provision of assistance in special cases ( $\$ 2$ AsylbLG analogous to SGB XII) reached a higher share of people entitled to benefits at the end of the year $(42.7 \%)$ than the minimum provision according to $\S 3$ AsylbLG (29.0\%). Nevertheless, it caused comparatively less gross expenditure.

Conclusion. It is recommended to apply $\$ 2$ AsylbLG for a prestay period of three months or longer in order to be able to grant assistance analogous to Chapters 5 to 9 SGB XII earlier. Nationwide introduction of an electronic health insurance card for asylum seekers would improve access.

Keywords

Asylum seekers - Refugees - Access to health care $\cdot$ Human rights psychischer, physischer oder sexueller Gewalt erlitten haben, wird die erforderliche medizinische oder sonstige Hilfe gewährt (\$6 Abs. 2 AsylbLG).

Die leistungsrechtlichen Merkmale Akutheit, Erforderlichkeit und Unaufschiebbarkeit zielen darauf, die Behandlung vor allem von chronischen Erkrankungen und ihre Versorgung mit Heil-, Hilfs- und Pflegemitteln auf die Zeit nach dem Anerkennungsverfahren oder der Abschiebung hinauszuzögern. Die harten Restriktionen in der Leistungsgewährung werden besonders deutlich bei der (Unter-)Versorgung mit Zahnersatz. Das belegen exemplarisch sozialmedizinische Auftragsgutachten des Medizinischen Dienstes der Krankenversicherung (MDK) Rheinland-Pfalz für den Öffentlichen Gesundheitsdienst, die bei 318 vorgelegten Behandlungsplanungen im Rahmen des AsylbLG nur rund ein Viertel für die Leistungsge- währung vollumfänglich empfahlen und über die Hälfte der Anträge komplett ablehnten [13].

Die Asylbewerberleistungsstatistik wird als Sekundärstatistik in Deutschland erhoben, bei der von den zuständigen Behörden vorliegende prozessgenerierte Verwaltungsdaten aufbereitet werden. Sie wird jährlich zum Stichtag 31.12. als Vollerhebung flächendeckend durchgeführt. Die Auskunftspflicht und die Erhebungsmerkmale sind in $\$ 12$ 
AsylbLG niedergelegt. Die Bundesstatistik berichtet die Zahl und Struktur der Leistungsberechtigten sowie die finanziellen Auswirkungen des AsylbLG. Die Daten werden in den statistischen Ämtern der Länder in einem gemeinsamen Aufbereitungsprogramm erfasst und nach Plausibilität überprüft. Qualitätsberichte informieren über die Methodik [14].

Für die vorliegende Arbeit wurden an einem Gastwissenschaftsarbeitsplatz im Forschungsdatenzentrum der Statistischen Ämter des Bundes und der Länder die beiden Scientific Use Files zu den „Empfängern von Asylbewerberregelleistungen“ und den „Empfängern von besonderen Asylbewerberleistungen" unter Geheimhaltungsvorschriften ausgewertet. Die „Statistik der Ausgaben und Einnahmen für Asylbewerberleistungen" steht nicht als Datensatz auf der Mikroebene, sondern in Tabellenform zur Verfügung. Die Statistiken weisen nur Personen mit bestehendem Leistungsbezug zum 31.12.2018 aus, während die Ein- und Ausgaben ganzjährig verbucht wurden. Personen, die vom BAMF die Zuerkennung der Flüchtlingseigenschaft erhalten haben oder als Asylberechtigte anerkannt sind, sind nicht mehr nach dem AsylbLG leistungsberechtigt. Die Bundesstatistiken werden alle 2 Jahre in der Fachserie 13 Reihe 7 publiziert [14]. $\mathrm{Zu}$ den für diese Auswertung relevanten Items zählen die Leistungsempfänger mit Geschlecht, Geburtsdatum, Staatsangehörigkeit, aufenthaltsrechtlichem Status und Wohnort. Es werden die Regelbedarfsstufen, Art und Träger der Unterkünfte sowie die gewährten Hilfearten und -formen erhoben. Bei den Empfängern von Asylbewerberregelleistungen werden darüber hinaus der Erwerbsstatus und der Leistungsbeginn registriert.

Die deskriptive Statistik und Korrelationsanalytik wurden mit der Statistiksoftware IBM SPSS Statistics 26.0 gerechnet.

\section{Ergebnisse}

Zum Stichtag 31.12.2018 haben 423.201 Personen in Deutschland Leistungen nach dem AsylbLG erhalten. $\bullet$ Tab. 1 führt die
Leistungsempfänger von Asylbewerberregelleistungen und ausschließlich besonderen Leistungen für einen Überblick zusammen. $64,1 \%$ der Leistungsempfänger sind Männer und 35,9\% Frauen (• Tab. 1, Sp. 2). Im Durchschnitt sind die Personen 24,2 Jahre alt $(\mathrm{SE}=0,03)$. Fast ein Drittel ist unter 18 Jahre alt. $53,9 \%$ stammen aus Asien mit den wichtigsten Herkunftsländern Afghanistan, Irak, Syrien, Iran und Pakistan. $23,1 \%$ sind Staatsangehörige von afrikanischen Ländern (Nigeria, Somalia etc.) und $20,0 \%$ von europäischen Ländern (Russische Föderation, Türkei etc.) Die Leistungsbezieher besitzen größtenteils eine Aufenthaltsgestattung (69,7\%) oder eine Duldung (17,3\%).

Von den Empfängern von Asylbewerberregelleistungen beziehen $44,2 \%$ die Unterstützung mit einer Dauer bis unter 15 Monaten (•Tab. 1, Sp. 2). Die Leistungsbezugsdauer beträgt im Mittel fast 2 Jahre bzw. 23,4 Monate ( $\mathrm{SE}=0,02)$.

$10,5 \%$ der Leistungsempfänger sind in Aufnahmeeinrichtungen untergebracht (• Tab. 1, Sp. 2). 40,7\% leben in Gemeinschaftsunterkünften und 48,8\% in dezentralen Unterbringungsformen (insbesondere Einzelwohnungen). Bei knapp einem Viertel der Leistungsempfänger sind die Länder als überörtliche Träger, bei dem Rest die Gemeinden und Kreise als örtliche Träger zuständig. Die Hälfte der Leistungsempfänger verteilt sich auf die 3 größten Bundesländer Nordrhein-Westfalen, Bayern und Baden-Württemberg.

An der Regelversorgung nach $\$ 2$ AsylbLG orientieren sich die analogen Leistungen entsprechend dem SGB XII, die unter den gesetzlichen Voraussetzungen den Leistungsberechtigten anstelle der Grundleistungen (nach 15 Monaten Voraufenthaltszeit) gewährt werden. Das betrifft die Hilfe zum Lebensunterhalt und die Leistungen in besonderen Fällen. Wie in $\bullet$ Tab. 2 (Sp. 1) gezeigt, erhielten von den Leistungsempfängern 79.480 ambulante Hilfe bei Krankheit, 3099 stationäre Hilfe bei Krankheit, 324 Hilfe bei Schwangerschaft und Mutterschaft, 1585 Hilfe zur Pflege und 3774 sonstige Hilfen als Analogleistungen ( $\$ 2$ AsylbLG) am Jahresende nach Kapitel 5-9 SGB XII.
- Tab. 2 informiert über die medizinische Minimalversorgung gemäß $\$ 3$ AsylbLG, nach dem zusätzlich zu den Grundleistungen im Bedarfsfall andere Leistungen nach $\$ \$ 4-6$ AsylbLG gewährt werden. Dementsprechend haben 62.151 Leistungsempfänger ambulante und 2237 stationäre Leistungen bei Krankheit, Schwangerschaft oder Geburt am Jahresende ( $\$ 4$ AsylbLG) erhalten (- Tab. 2, Sp. 1). Hinzu kommen für 12.080 Leistungsempfänger sonstige Leistungen in Form von Sach- und/oder Geldmitteln ( $\$ 6$ AsylbLG).

Diese Hilfearten werden für die Leistungsbezieher zum 31.12. sowohl stichtagsbezogen als auch für den gesamten Jahresverlauf registriert (• Tab. 2). Wie in Spalte 2 ausgewiesen, ist der Anteil der Leistungsempfänger von ambulanten Leistungen bei Krankheit, Schwangerschaft oder Geburt dann in der Jahresbetrachtung mehr als doppelt und von stationären Behandlungsformen fast 7-mal so hoch.

Die Minimalversorgung und die Regelversorgungkönnen anhand der jeweils gewährten Hilfen zur Gesundheit in der Reichweite direkt verglichen werden:

Im Bereich der Minimalversorgung gemäß \$3 AsylbLG haben 66.654 Personen am Jahresende 2018 eine Leistung bei Krankheit ( $\$ 4$ AsylbLG) und/oder eine sonstige Leistung ( $\$ 6$ AsylbLG) in Anspruch genommen. Bezogen auf die leistungsberechtigte Grundgesamtheit von 229.818 Personen (• Tab. 3, Sp. 10-11) kann eine Inanspruchnahmequote von $29,0 \%$ am Jahresende bestimmt werden. Dem steht die gesundheitsbezogene Regelversorgung ( $\$ 2$ AsylbLG) analog dem SGB XII gegenüber. Hier wurden am Jahresende 82.579 Leistungsempfängern ambulante oder stationäre Hilfe bei Krankheit gewährt. Das entspricht bei einer leistungsberechtigten Grundgesamtheit von 193.383 Personen (• Tab.3, Sp. 8-9) einer wesentlich höheren Inanspruchnahme von $42,7 \%$. Der direkte Vergleich zeigt im Ergebnis, dass von der wohlfahrtsstaatlich großzügigeren Regelung ein höherer Prozentsatz unter den Leistungsberechtigten profitiert hat (42,7\% vs. $29,0 \%)$.

Gleichzeitig demonstriert die Gegenüberstellung in - Tab. 4 (Spalten 8 vs. 


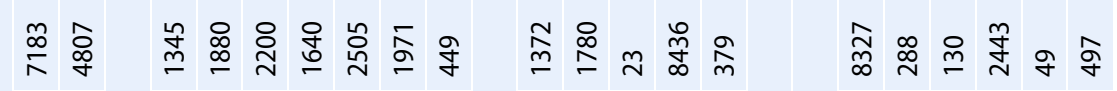

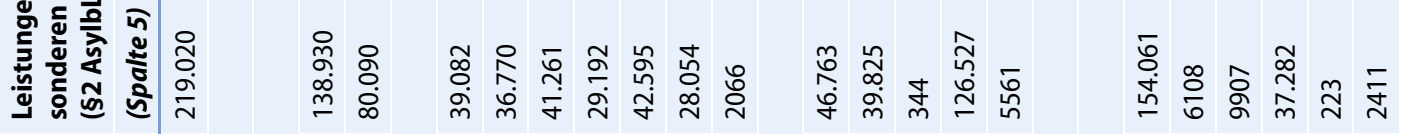
.

$\sqrt{1}$

II

客
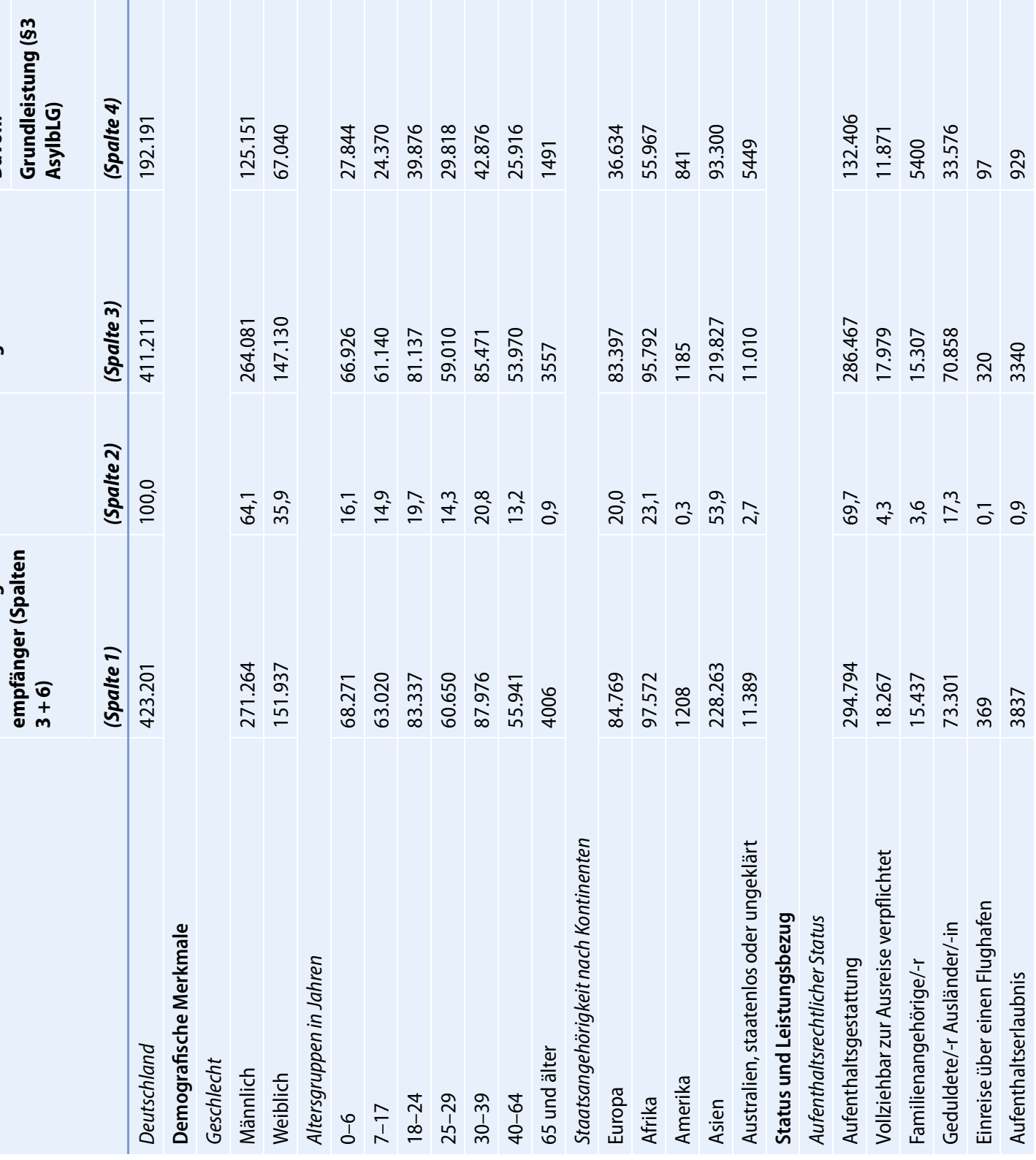


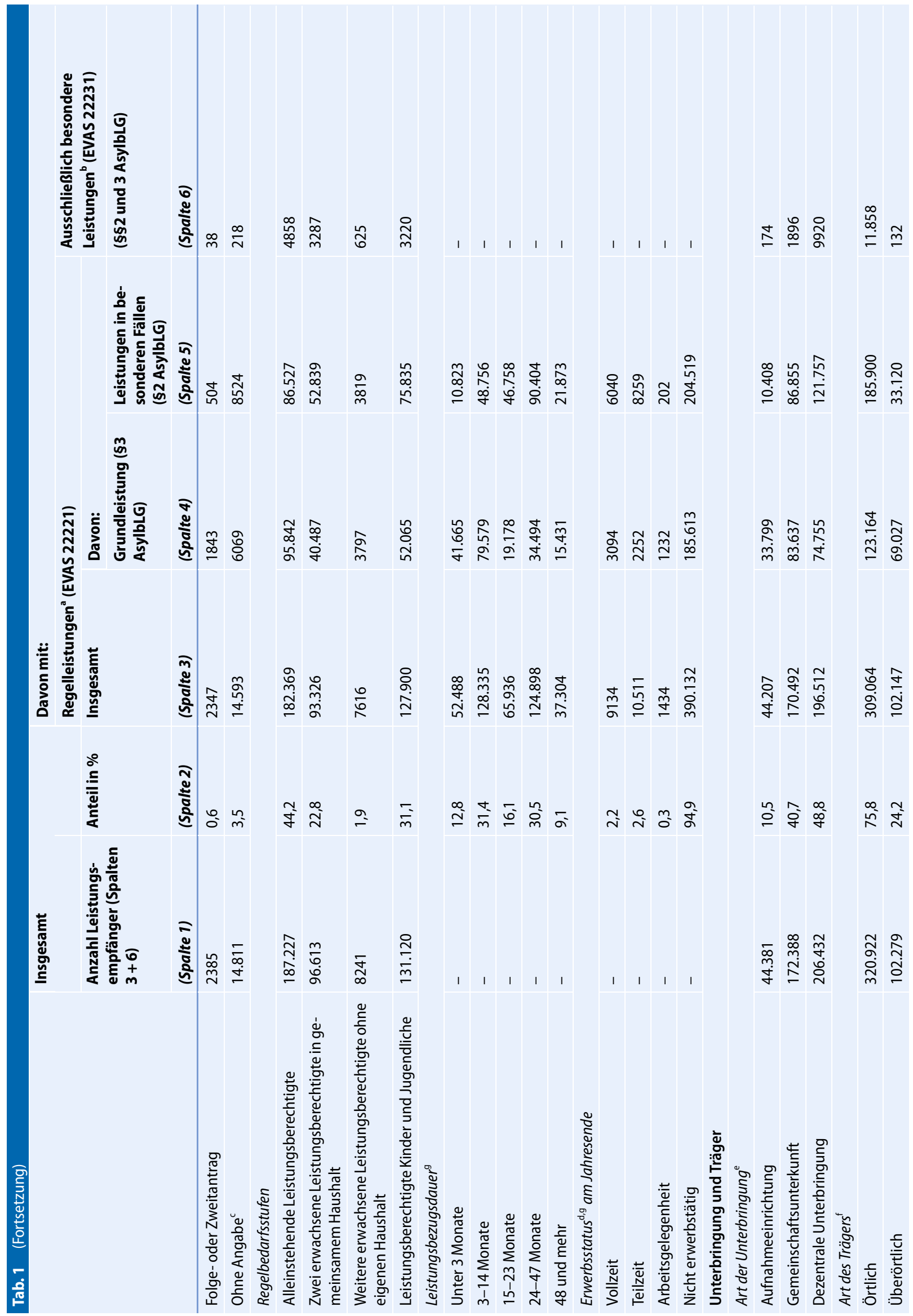




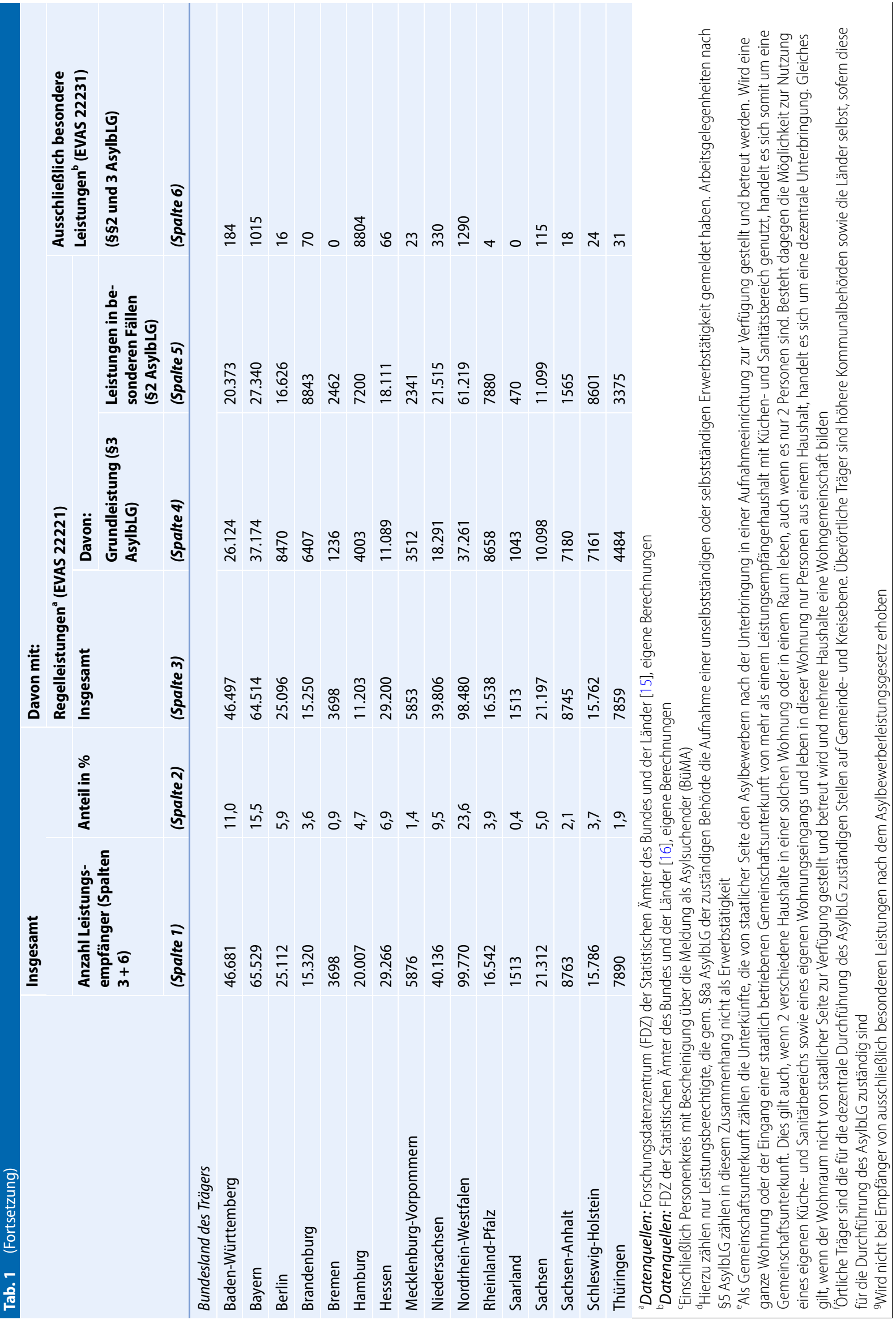




\section{Originalien und Übersichten}
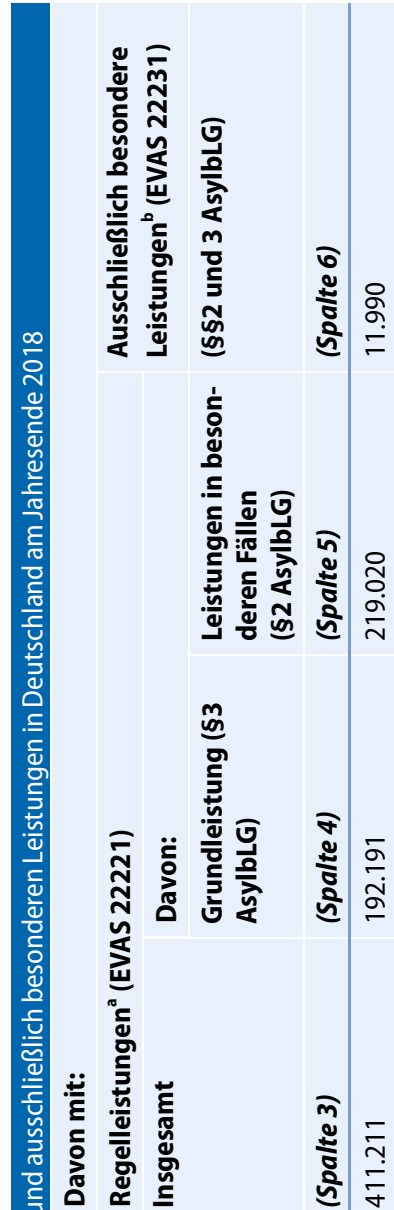<smiles>[CH][CH]</smiles>

๕ั
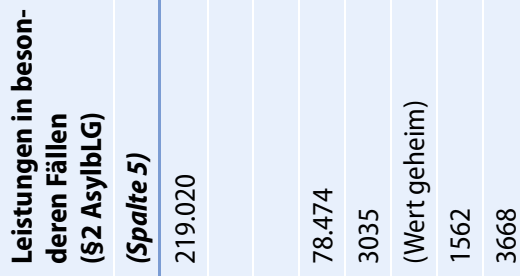

$\underset{\bar{m}}{\stackrel{\Sigma}{\sim}} \stackrel{\infty}{\sim}$

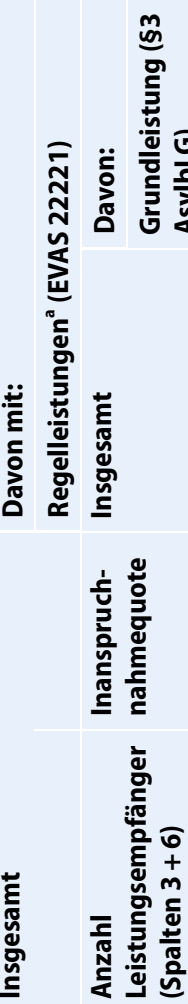

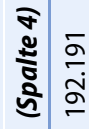

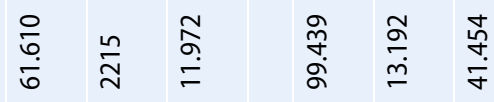

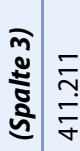

$+\frac{\bar{\varepsilon}}{\bar{d}}$

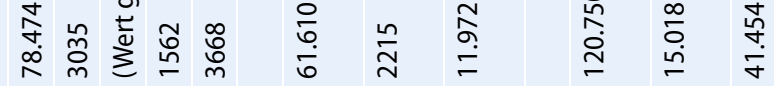

ฟิ

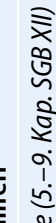

产

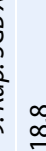

冓

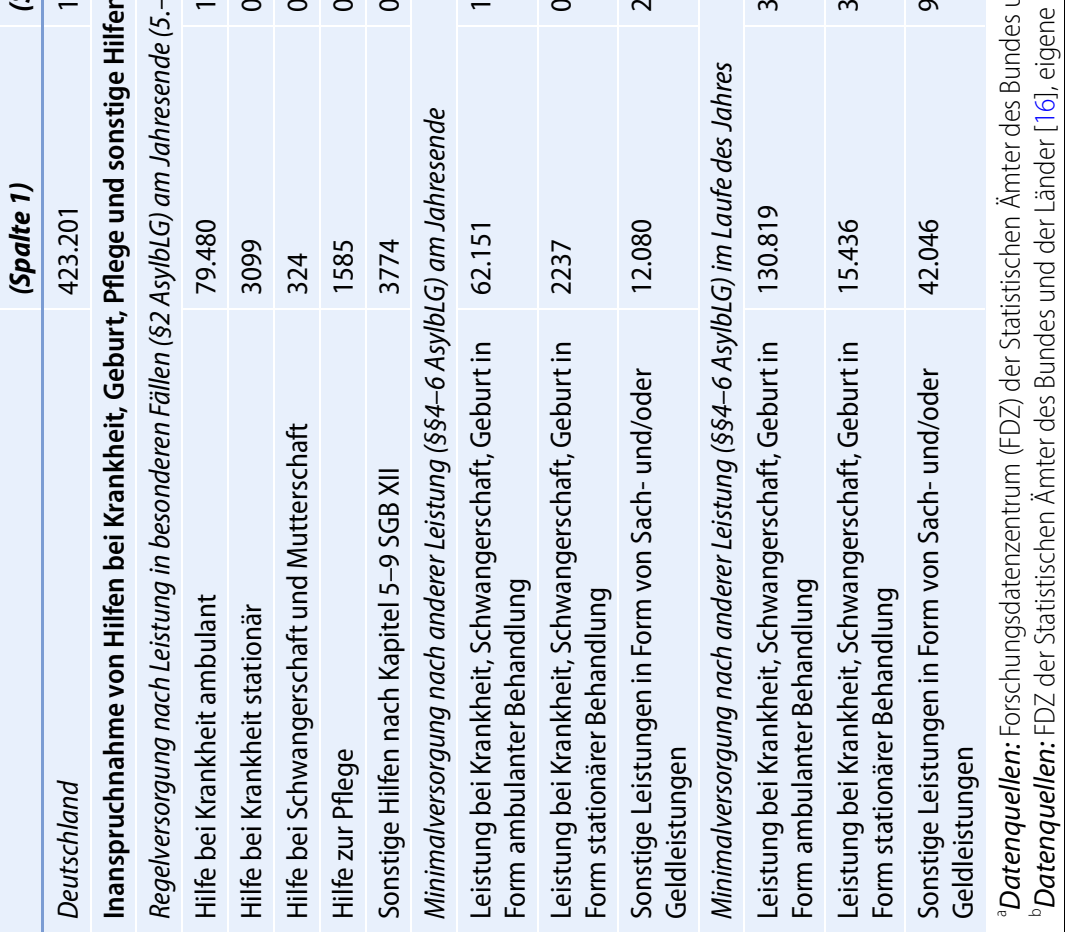




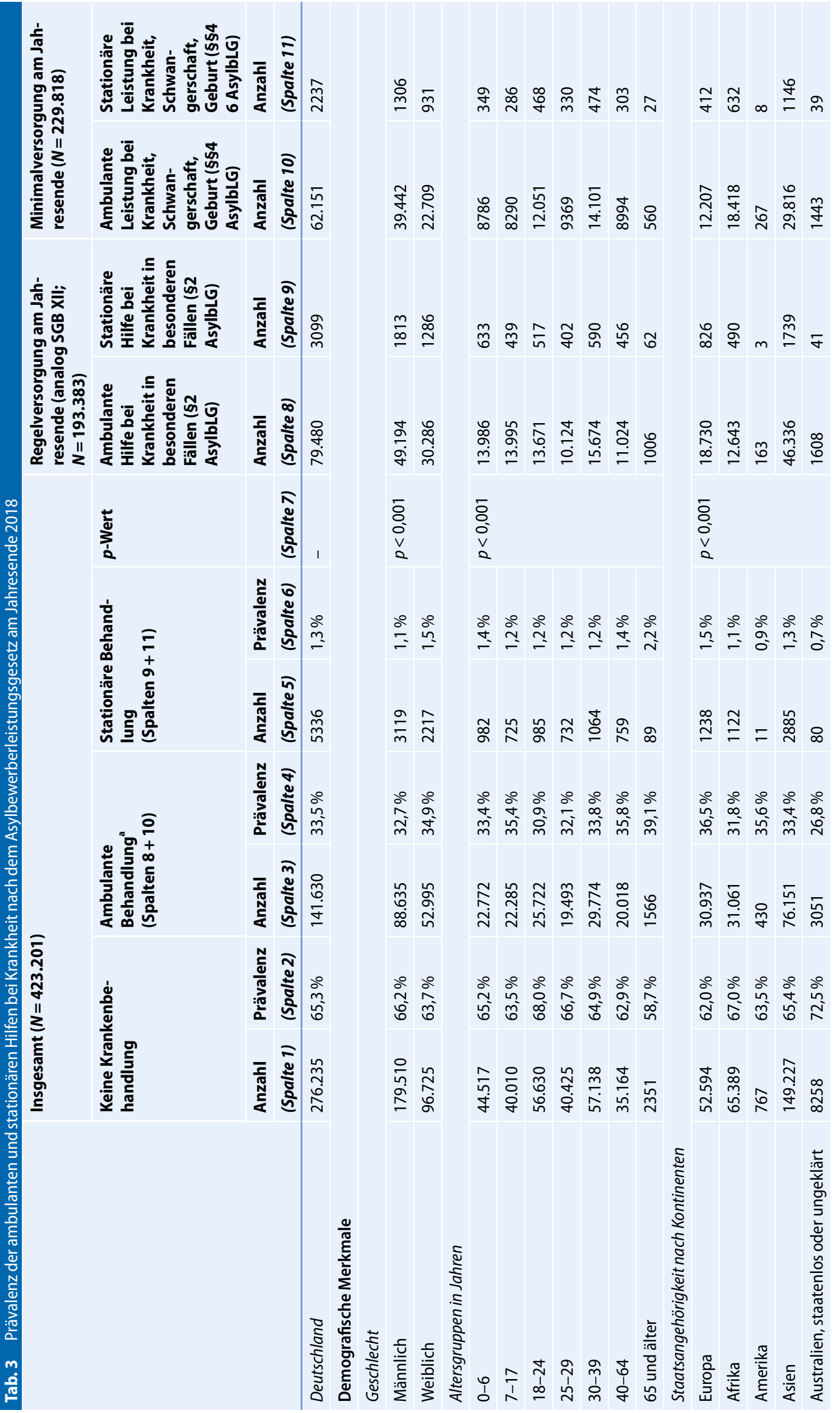




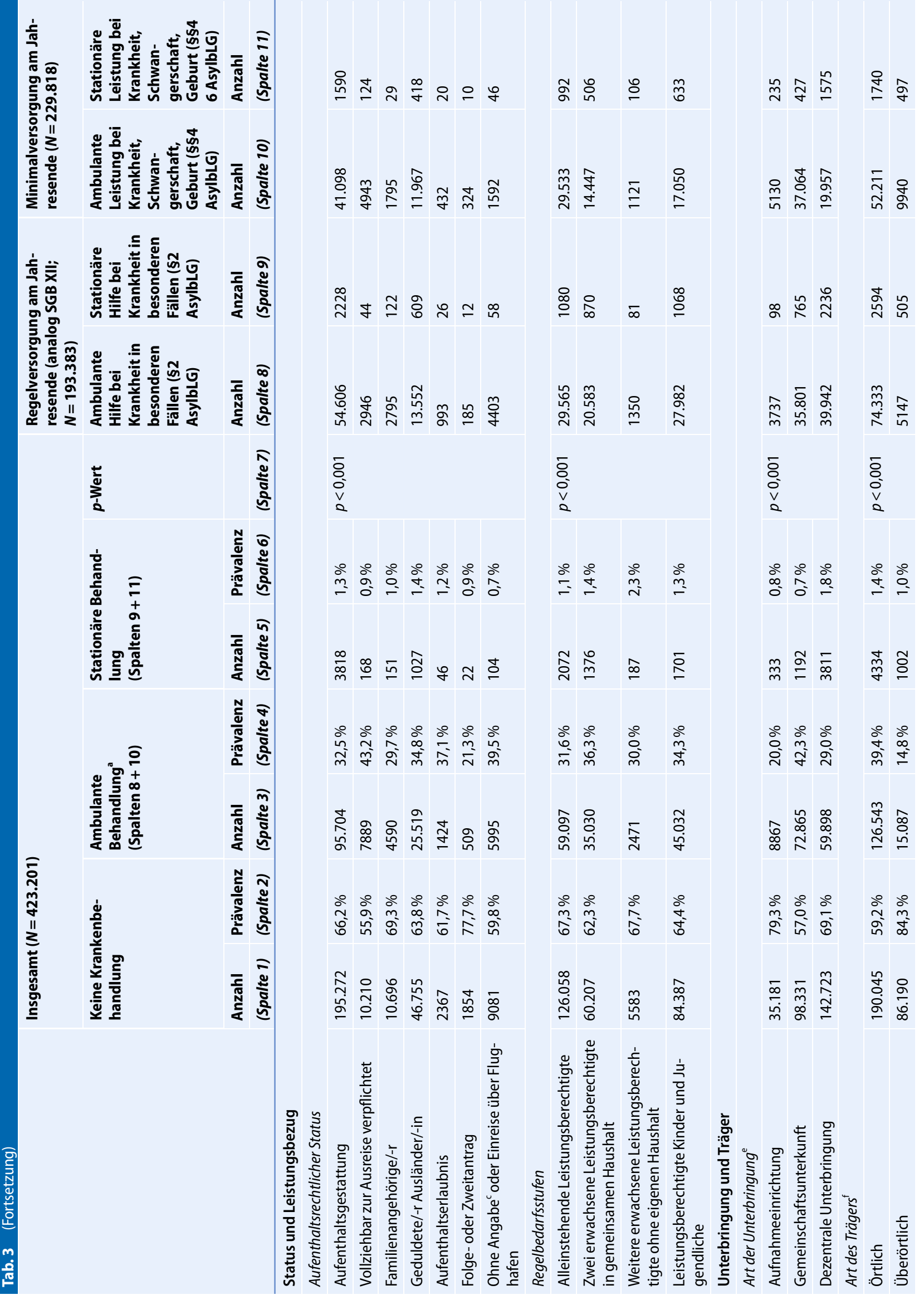




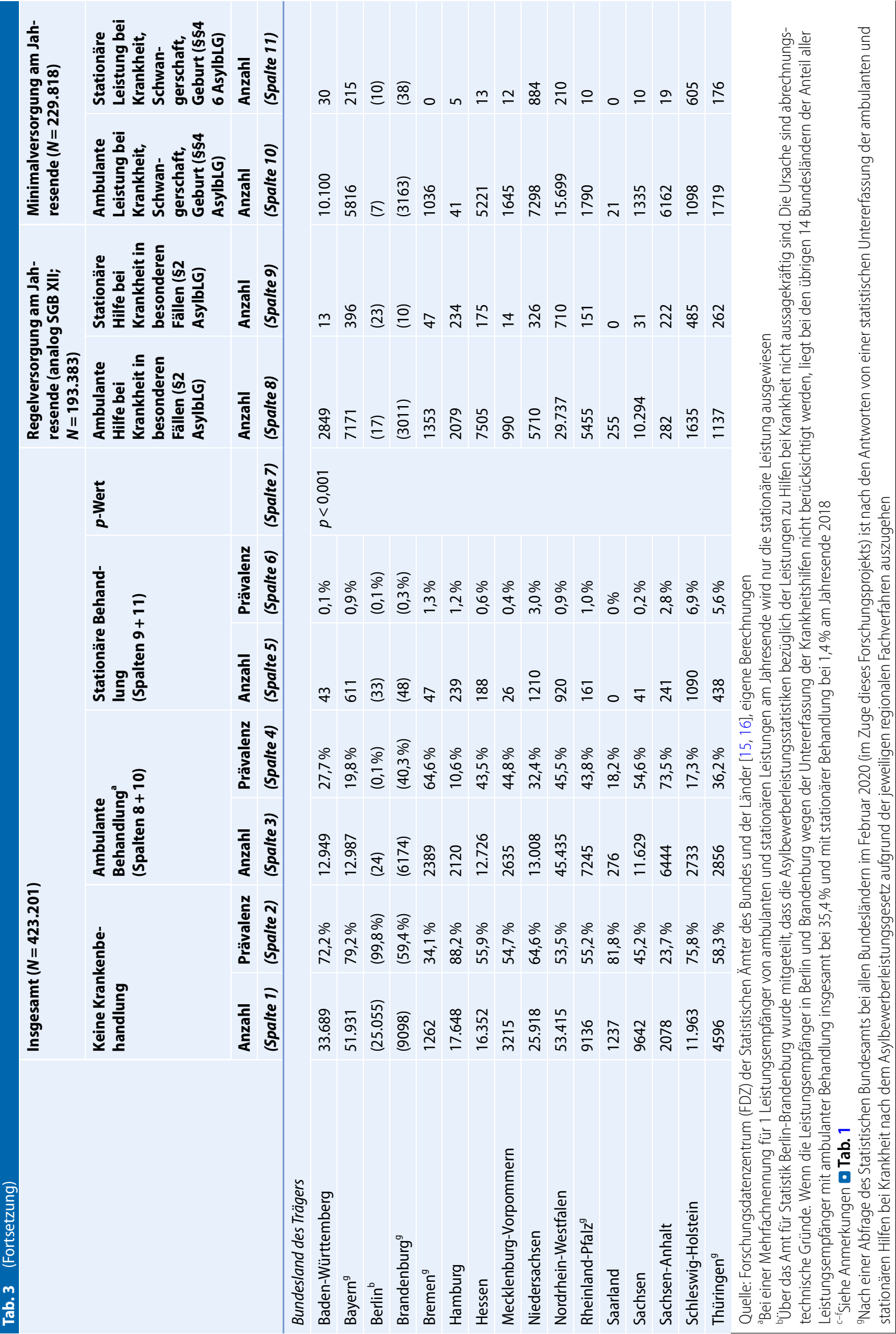




\section{Originalien und Übersichten}

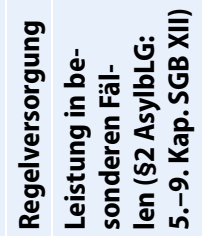

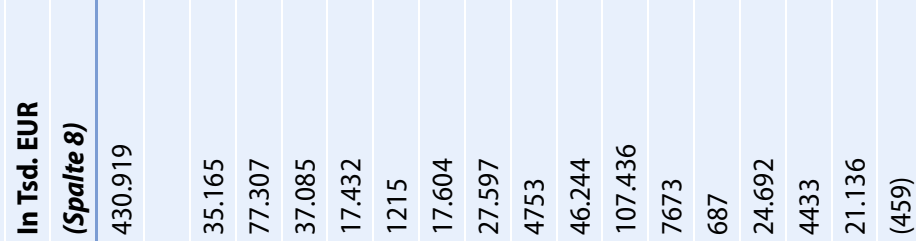

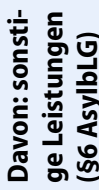

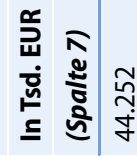

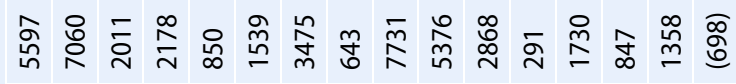

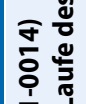

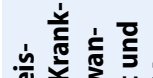

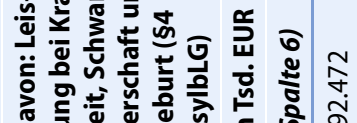

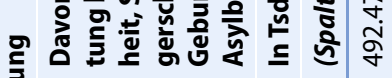

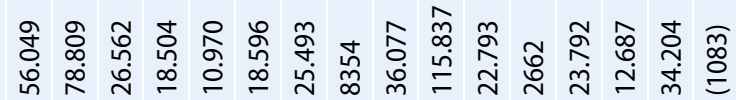
ㅎํำ

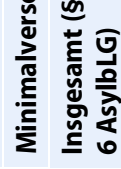

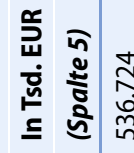

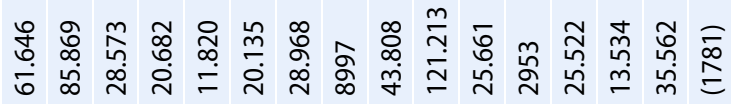

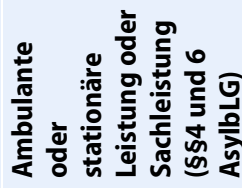

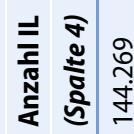

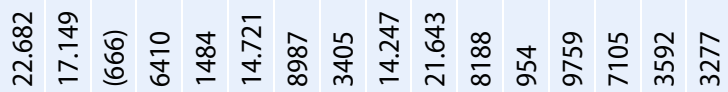

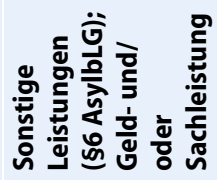

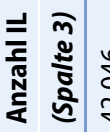

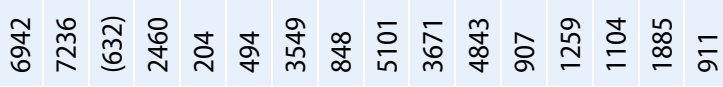
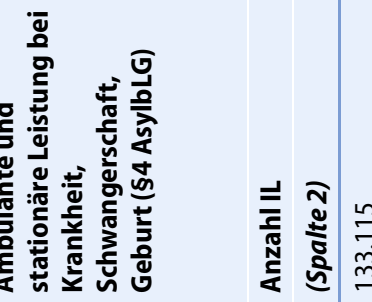

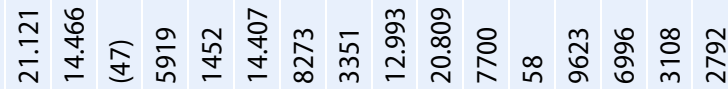

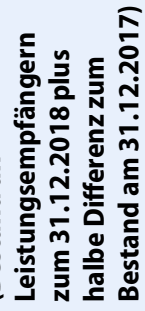

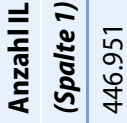

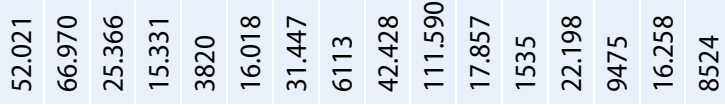

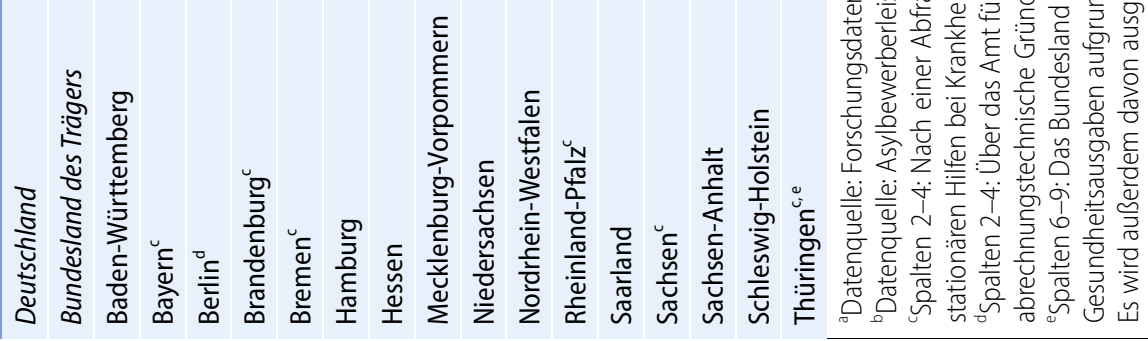




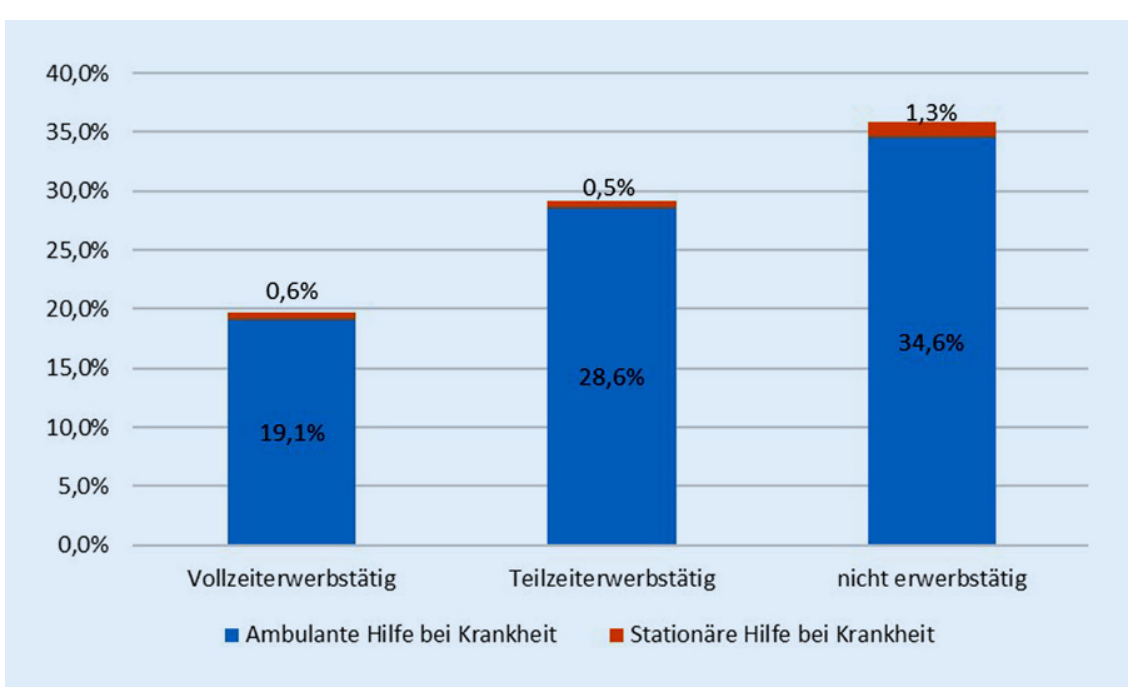

Abb. 1 A Inanspruchnahme der Hilfen bei Krankheit nach Erwerbsstatus am Jahresende 2018. Quelle: Forschungsdatenzentrum (FDZ) der Statistischen Ämter des Bundes und der Länder [15], eigene Berechnung und Darstellung. $(N=411.211)$

5), dass die Regelversorgung mit analogen gesundheitsrelevanten Hilfen in besonderen Fällen ( $\$ 2$ AsylbLG analog SGB XII) nicht nur mehr leistungsberechtigte Menschen in Deutschland erreicht, sondern bei den Bruttoausgaben insgesamt weniger Kosten als die bürokratieaufwendige Minimalversorgung nach $\$ 3$ AsylbLG verursacht hat.

- Tab. 3 kombiniert die beiden Hilfearten bei Krankheit nach $\$ \$ 2$ und 3 AsylbLG. Für alle Leistungsempfänger werden gemeinsame Prävalenzraten sowohl der ambulanten als auch der stationären Krankenversorgung berechnet. Zum Stichtag 31.12.2018 befanden sich demnach 33,5\% aller Leistungsempfänger in ambulanter Behandlung und 1,3\% in stationärer Behandlung. Die Prävalenzraten variieren innerhalb der soziodemografischen Gruppen in $\mathbf{0}$ Tab. 3 in statistisch signifikanter Weise und sind bei den Frauen, Älteren und Angehörigen europäischer Staaten leicht erhöht. Auffällig hoch ist der Anteil mit ambulanter Krankenversorgung in der Personengruppe, die vollziehbar zur Ausreise verpflichtet ist.

Zum Schutz der Bevölkerung werden nach $\$ 62$ Asylgesetz (AsylG) alle Ausländer, die in einer Aufnahmeeinrichtung oder Gemeinschaftsunterkunft $\mathrm{zu}$ wohnen haben, verpflichtet, eine ärztliche Untersuchung auf übertragbare Krankheiten einschließlich einer Rönt- genaufnahme der Atmungsorgane zu dulden. Der Öffentliche Gesundheitsdienst ist angehalten, den Impfstatus von Asylsuchenden systematisch zu kontrollieren und mit freiwilligen Angeboten auf die Schließung von Impflücken gemäß den Empfehlungen der Ständigen Impfkommission (STIKO) frühzeitig hinzuwirken [18]. Speziell für die Aufnahmeeinrichtungen, die in der $\mathrm{Zu}$ ständigkeit der Länder liegen, zeigt sich hier (entgegen den Erwartungen) kein Sondereffekt (siehe $\bullet$ Tab. 3). In den Aufnahmeeinrichtungen sowie generell bei überörtlichen Trägern gibt es eine weit unterdurchschnittliche Gewährung von ambulanten oder stationären Leistungen bei Krankheit.

4,8\% der Empfänger von Asylbewerberregelleistungen sind erwerbstätig (davon 89,3\% Männer). - Abb. 1 veranschaulicht eine enge Assoziation zwischen Krankheitshilfen und der Arbeitsmarktintegration. Erwartungsgemäß ist der Anteil mit ambulanten oder stationären Leistungen bei Krankheit unter den Empfängern von Asylbewerberregelleistungen in Erwerbstätigkeit signifikant geringer als in Nichterwerbstätigkeit am Jahresende (Chi-Quadrat $=1237,4$; $\mathrm{df}=4 ; p<0,001)$.

Am Jahresende 2018 ist bei den verschiedenen Bundesländern eine außerordentlich große Bandbreite hinsichtlich der Gewährung von Leistungen bei
Krankheit $\mathrm{zu}$ beobachten. Sie reicht von $11,8 \%$ der Leistungsempfänger in Hamburg bis in der Spitze zu 76,3\% in Sachsen-Anhalt (• Tab. 3, Sp. 4+6).

- Abb. 2 basiert auf den amtlichen Angaben zu den Bruttoausgaben für den gesamten Jahresverlauf (• Tab. 4, Sp. 5-8). Nachdem die Leistungsempfänger nur punktuell zum 31.12. erhoben werden, wird als Bezugsgröße der Jahresdurchschnitt für eine Näherung geschätzt. Der Vorjahresbestand zum 31.12.2017 war nach der Statistik in allen Bundesländern höher und nahm jeweils prozentual verschieden bis zum 31.12.2018 ab. Die Differenz zwischen den Beständen am Beginn und Ende 2018 wird daher mit der Hälfte für einen kalkulatorischen Jahresdurchschnitt auf der Bundesländerebene berücksichtigt (• Tab. 4, Sp. 1).

Nach dieser groben Kalkulation liegen die Bruttoausgaben für Leistungen in besonderen Fällen ( $\$ 2$ AsylbLG) oder bei Krankheit, Schwangerschaft, Geburt ( $\$ 4$ AsylbLG) und/oder sonstige Leistungen ( $\$ 6$ AsylbLG) pro Kopf im Jahresdurchschnitt bei insgesamt $2165 €$ in Deutschland. - Abb. 2 illustriert, dass die Länder Schleswig-Holstein und Bremen auf dieser Berechnungsgrundlage mit weitem Abstand die höchsten ProKopf-Bruttoausgaben haben (3487€ und $3413 €$ ), während am anderen Pol in Hessen lediglich etwas über der Hälfte verausgabt wurde.

Die 4 (kleinen) Bundesländer mit den höchsten Pro-Kopf-Bruttoausgaben für Gesundheitsleistungen haben eine Landesrahmenvereinbarung nach $\$ 264$ SGB V für die Gesundheitskarte abgeschlossen. Die Bundesländer mit Gesundheitskarte weisen eine signifikant höhere Inanspruchnahmequote von stationären Leistungen am Jahresende als die übrigen Bundesländer mit 1,7\% vs. $0,6 \%$ auf (Chi-Quadrat $=968,2 ; \mathrm{df}=2$; $p<0,001)$. Bei der ambulanten Versorgung gibt es dagegen kaum Unterschiede.

Eine Heterogenität im Leistungsgeschehen zeigt sich nicht nur zwischen den Bundesländern, sondern auch zwischen den Kreisen, die aber aus Geheimhaltungsgründen nicht dargestellt werden kann. So wurden in 17 Kreisen für die gemeldeten Leistungsempfänger am Jahresende überhaupt keine ambulanten 


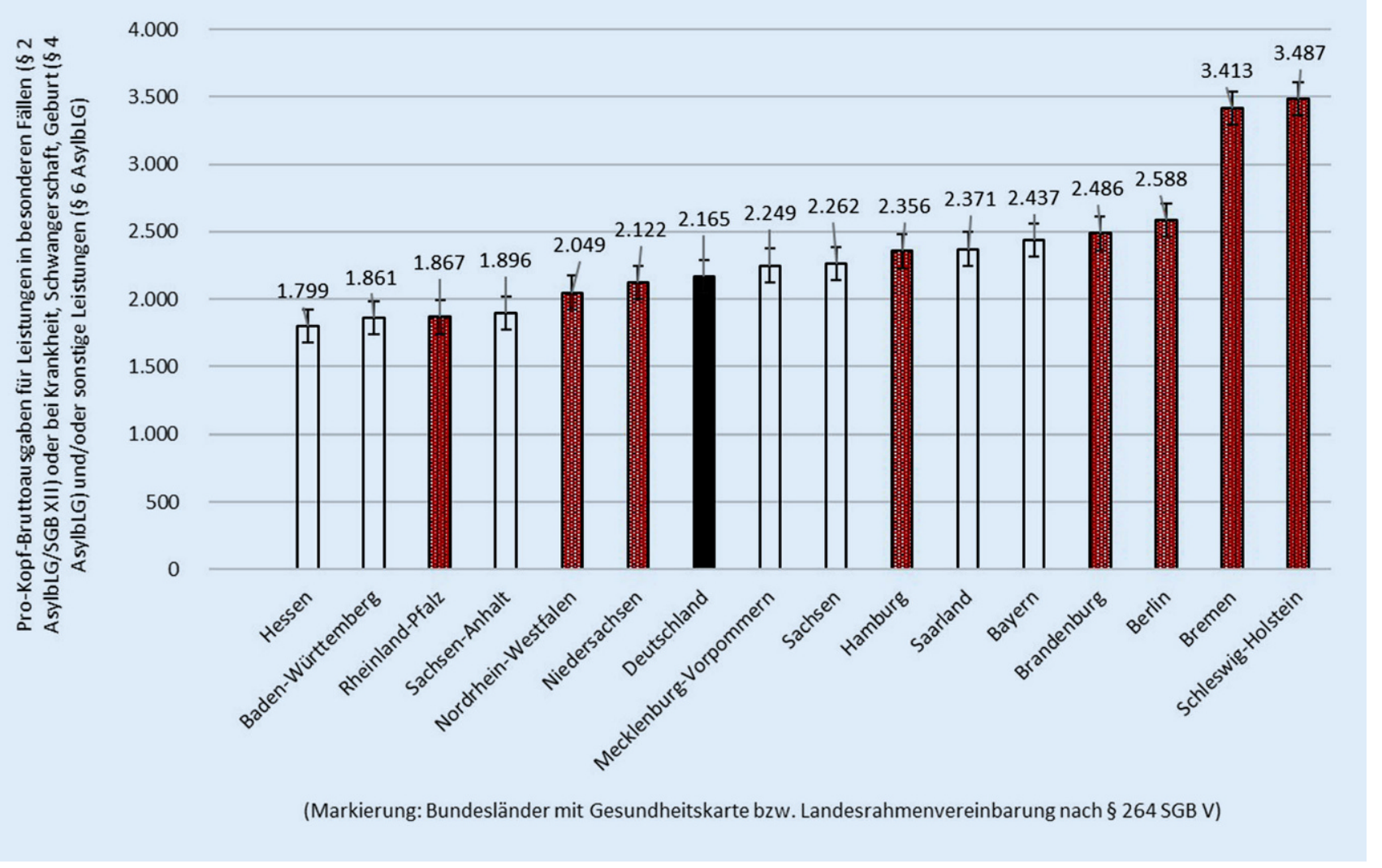

Abb. $2 \Delta$ Gesundheitsausgaben je Leistungsberechtigten gemäß Asylbewerberleistungsgesetz in den Bundesländern im Jahresdurchschnitt 2018. Datenquellen: Forschungsdatenzentrum (FDZ) der Statistischen Ämter des Bundes und der Länder [15-17]. Eigene Berechnungen und Darstellung. Anmerkungen: 1. Das Bundesland Thüringen wird in • Abb. 2 nicht separat ausgewiesen, weil es nach einer Länderabfrage des Statistischen Bundesamts im Februar 2020 (im Zuge dieses Forschungsprojekts) die Gesundheitsausgaben aufgrund des regionalen Fachverfahrens nach eigenen Aussagen nicht valide statistisch erfasst. 2. Es wird außerdem davon ausgegangen, dass die verwaltungsmäßigen Schwierigkeiten bei den Jahresabgrenzungen in den Rechnungsstellungen alle Bundesländer gleich betreffen

oder stationären Behandlungsfälle angegeben.

\section{Diskussion und Fazit}

Die Asylbewerberstatistik ist als jährliche Vollerhebung am Jahresende angelegt und das AsylbLG verpflichtet, über die Erhebungsmerkmale Auskunft zu geben. Dabei sind methodische Limitationen zu beachten. Da der objektive medizinische Versorgungsbedarf (z.B. durch Screenings) bei asylsuchenden Menschen nicht statistisch erhoben wird, bestehen Limitationen bei der Interpretation der unterschiedlichen Inanspruchnahmen von Krankheitshilfen zwischen den Gruppen oder Regionen. Ein Bias entsteht, wenn die Berichtsstellen einzelne Fälle bzw. Hilfen nicht oder nachträglich erfassen. Nach der berichteten Abfrage des Statistischen Bundesamts im Februar
2020 ist von einer statistischen Untererfassung der ambulanten und stationären Hilfen bei Krankheit nach dem Asylbewerberleistungsgesetz in verschiedenen Bundesländern auszugehen (vgl. Anmerkungen bei $\bullet$ Tab. 3-4 und $\bullet$ Abb. 1-2). Der Vergleich zwischen den Bundesländern offenbart eine große Heterogenität im ambulanten und stationären Leistungsgeschehen bei Krankheit und muss daher besonders vorsichtig interpretiert werden. In den bevölkerungsstarken Bundesländern wie in Nordrhein-Westfalen oder Baden-Württemberg scheint die Erfassung nach den Rückmeldungen wesentlich besser zu funktionieren, was auch weniger statistische Unsicherheiten für die Bundesdurchschnittswerte bedeutet. Eine zeitliche Jahresabgrenzung ist in der offiziellen Statistik und in der Software jedoch für die Ausgabenrechnung generell nicht vorgesehen.
Die Leistungsempfänger werden nur im Sinne einer Bestandsstatistik zum 31.12. erfasst. Für weitergehende Analysen fehlen Zugangs- und Abgangsstatistiken im Jahreszeitraum. Die Ein- und Ausgabenrechnung bezieht sich dagegen auf alle Verbuchungen im Jahresverlauf. Methodisch lassen sich daher bei den Verknüpfungen zwischen den Bruttoausgaben und den Leistungsempfängern nach Hilfearten nur Näherungswerte eruieren. Wünschenswert wäre in der Zukunft die Zuordnung der Bruttoausgaben auf der Personenebene und bei den Krankheitshilfen mindestens die Registrierung der Hauptdiagnosegruppe. Hier besteht weiterer Forschungsbedarf.

In der wissenschaftlichen Perspektive liegen auf der einen Seite die Probleme bei den Asylbewerberleistungsstatistiken als Datenquelle für die Gesundheitsversorgung in der Erhebungskonzeption und 
in der Untererfassung in einigen Bundesländern. Die offiziellen Fachserien und Qualitätsberichte negieren bis dato die Einflüsse auf die Qualität der Gesundheitsdaten (vgl. [14]). Der Zweck dieser Erhebungen als Bundesstatistik ist nach $\$ 12$ AsylbLG, die „Auswirkungen dieses Gesetzes und zu seiner Fortentwicklung zu beurteilen“. Für den Gesundheitsbereich müsste dafür die Datenbasis weiterentwickelt werden. Eine große Chance läge im Aufbau einer analogen Gesundheitsberichterstattung über die Routinen in der gesetzlichen Krankenversicherung für die Bundesländer, die die Gesundheitskarte für Asylsuchende bereits eingeführt haben.

Auf der anderen Seite haben das Erhebungsinstrument und die Forschungsdatensätze große methodische Vorzüge. Alleinstellungsmerkmale sind die flächendeckende Vollerhebung der Leistungsempfänger nach dem AsylbLG mit gesetzlicher Auskunftspflicht und die jährliche Wiederholung identischer Items. Mit der vorliegenden Studie werden erstmals die Forschungsdatensätze mit den gewährten ambulanten und stationären Leistungen bei Krankheit nach dem AsylbLG umfassend wissenschaftlich analysiert. Bisher werden ambulante und stationäre Krankheitshilfen in den Bundesstatistiken nicht separat ausgewiesen. Die Auswertungen geben nicht nur Einblick in die unterschiedlichen Inanspruchnahmen von Krankheitshilfen bei den Leistungsberechtigten nach soziodemografischen Gruppen, sondern auch nach Status, Leistungsbezug und Unterbringungsart. So werden in den Aufnahmeeinrichtungen und im $\mathrm{Zu}$ ständigkeitsbereich von überörtlichen Trägern relativ wenig Krankheitshilfen bei den Leistungsempfängern am Jahresende 2018 registriert. Das ist bemerkenswert, weil mit anderen Datenquellen festgestellt wurde, dass Flüchtlinge in derartigen Einrichtungen häufiger unter psychischen Erkrankungen als in dezentralen Wohnformen leiden [8]. Die großen Fallzahlen lassen auch Analysen zu kleinen Statusgruppen wie die vollziehbar zur Ausreise verpflichteten Leistungsempfänger (mit hoher Inanspruchnahme von Krankheitshilfen) zu. Die Auswertung zeigt eine höhere Inan- spruchnahme von Krankheitshilfen bei den nichterwerbstätigen Asylsuchenden im Vergleich zu Teilzeit- und Vollzeitbeschäftigten. Das bestätigt eine frühere Befragung bei Flüchtlingen, die höhere psychische Disstresswerte in Erwerbslosigkeit ergab [19]. Am größten ist die Heterogenität bei der Gewährung von Leistungen bei Krankheiten allerdings zwischen den Bundesländern. Das deckt sich mit den von Razum et al. [10] sowie von Wächter-Raquet [5] beschriebenen bundesweit uneinheitlichen Gesundheitsversorgungsstrukturen bei Asylsuchenden.

Die Prävalenzraten speziell in der stationären Krankenversorgung variieren sowohl zwischen der Minimal- und Regelversorgung nach $\$ \$ 2$ und 3 AsylbLG als auch zwischen den Bundesländern mit und ohne Gesundheitskarte beträchtlich (• Tab. 3). Nachdem die Einweisung in ein Krankenhaus durch den behandelnden Arzt oder die Krankenhausambulanz erfolgt, sind diese regionalen Befunde ein Indiz für eine medizinisch nicht bedarfsgerechte Versorgung von asylsuchenden Menschen. Die Indizien legen nahe, dass die Gesundheitskarte den Zugang in die Krankenhausversorgung erleichtert, was auch die höheren ProKopf-Ausgaben in Bremen und Schleswig-Holstein erklären dürfte (• Abb. 2) und konform zu Länderberichten von Wächter-Raquet [5] ist. Die frühere Behandlung von schwerwiegenden Erkrankungen könnte den Heilungsverlauf in der Prognose positiv beeinflussen und das Risiko für spätere Folgekosten aufgrund von Verchronifizierung etc. absenken. Eine offene Forschungsfrage ist, welche Einflussnahme Zuzahlungen (z.B. Krankenhaustagegeld) in Bezug auf die Inanspruchnahmen in dieser Zielgruppe ausüben.

Aus diesen Diskussionssträngen lassen sich zwei Hauptempfehlungen ableiten:

1. Die Anwendung des $\$ 2$ AsylbLG schon bei einer Voraufenthaltszeit ab 3 Monaten, um frühzeitiger und bei mehr Leistungsberechtigten die Leistungen analog zu Kap. 5-9 SGB XII gewähren zu können. Die obigen Ergebnisse deuten an, dass diese Variante nicht nur bedarfsgerechter, sondern insgesamt kosteneffizienter für Deutschland ist. Das steht auch in Übereinstimmung mit Bozorgmehr und Razum [3].

2. Die flächendeckende Einführung der Gesundheitskarte in Deutschland nach einheitlichen Standards.

Die beiden Maßnahmen würden nicht nur dem Anspruch der Universalität der Menschenrechte und dem ungedeckten medizinischen Bedarf der Asylsuchenden gerecht werden, sondern auch dazu beitragen, die Gesundheitsversorgung für Asylsuchende $\mathrm{zu}$ vereinheitlichen und die im AsylbLG schon angelegte Zweiklassenmedizin in Deutschland $\mathrm{zu}$ überwinden. Darüber hinaus würden sie zur Entbürokratisierung im Gesundheitswesen und Entlastung des Öffentlichen Gesundheitsdienstes beitragen. Auf den durch die Gesundheitskarte generierten GKV-Routinedaten könnten dann auch die Gesundheitsforschung und die Bundesstatistik für das AsylbLG einheitlich und flächendeckend aufbauen. Die aktuelle Gesundheitskrise durch die SARS-CoV-2-19-Pandemie verschärft die Situation von Flüchtlingen gerade in den Aufnahme- und Gemeinschaftsunterkünften und erfordert ein schnelles Handeln [20].

\section{Korrespondenzadresse}

\section{Prof. Dr. Alfons Hollederer}

Fachbereich 01 Humanwissenschaften, Professur Theorie und Empirie des Gesundheitswesens, Universität Kassel Arnold-Bode-Str. 10 (WISO C), 34109 Kassel, Deutschland

alfons.hollederer@uni-kassel.de

Funding. Open Access funding provided by Projekt DEAL.

\section{Einhaltung ethischer Richtlinien}

Interessenkonflikt. A. Hollederer erklärt, dass kein wirtschaftlicher oder persönlicher Interessenkonflikt vorliegt. A. Hollederer erklärt, dass er keine finanzielle Förderung erhalten hat.

Für die vorliegende Arbeit wurden Sekundärdaten der Statistischen Ämter des Bundes und der Länder verarbeitet und die ethischen, rechtlichen und behördlichen Normen und Standards eingehalten. 
Open Access. Dieser Artikel wird unter der Creative Commons Namensnennung 4.0 International Lizenz veröffentlicht, welche die Nutzung, Vervielfältigung, Bearbeitung, Verbreitung und Wiedergabe in jeglichem Medium und Format erlaubt, sofern Sie den/die ursprünglichen Autor(en) und die Quelle ordnungsgemäß nennen, einen Link zur Creative Commons Lizenz beifügen und angeben, ob Änderungen vorgenommen wurden.

Die in diesem Artikel enthaltenen Bilder und sonstiges Drittmaterial unterliegen ebenfalls der genannten Creative Commons Lizenz, sofern sich aus der Abbildungslegende nichts anderes ergibt. Sofern das betreffende Material nicht unter der genannten Creative Commons Lizenz steht und die betreffende Handlung nicht nach gesetzlichen Vorschriften erlaubt ist, ist für die oben aufgeführten Weiterverwendungen des Materials die Einwilligung des jeweiligen Rechteinhabers einzuholen.

Weitere Details zur Lizenz entnehmen Sie bitte der Lizenzinformation auf http://creativecommons.org/ licenses/by/4.0/deed.de.

\section{Literatur}

1. Eichenhofer $E$ (2013) Gesundheitsleistungen für Flüchtlinge. ZAR 33(5-6):169-175. https://www. zar.nomos.de/fileadmin/zar/doc/Aufsatz_ZAR_ 13_5-6.pdf

2. Bundesärztekammer (2013) Stellungnahme der Zentralen Kommission zur Wahrung ethischer Grundsätze in der Medizin und ihren Grenzgebieten (Zentrale Ethikkommission) bei der Bundesärztekammer „Versorgung von nicht regulär krankenversicherten Patienten mit Migrationshintergrund “.'Dtsch Arztebl 110(18):899-902

3. Bozorgmehr K, Razum O (2015) Effect of restricting access to health care on health expenditures among asylum-seekers and refugees. A quasiexperimental study in Germany, 1994-2013. PLoS ONE 10(7):1-22. https://doi.org/10.1371/journal. pone. 0131483

4. Nationale Akademie der Wissenschaften Leopoldina (2015) Zur Gesundheitsversorgung von Asylsuchenden. Kurz-Stellungnahme

5. Wächter-Raquet M (2016) Einführung der Gesundheitskarte für Asylsuchende und Flüchtlinge. Der Umsetzungsstand im Überblick der Bundesländer. Gütersloh. https://www. bertelsmann-stiftung.de/fileadmin/files/BSt/ Publikationen/GrauePublikationen/Studie_VV Gesundheitskarte_Fluechtlinge_2016.pdf

6. Bozorgmehr K, Nöst S, Thaiss HM, Razum O (2016) Die gesundheitliche Versorgungssituation von Asylsuchenden. Bundesweite Bestandsaufnahme über die Gesundheitsämter. Bundesgesundheitsblatt 59(5):545-555. https://doi.org/10.1007/ s00103-016-2329-4

7. Frank L, Yesil-Jürgens R, Razum $O$ et al (2017) Gesundheit und gesundheitliche Versorgung von Asylsuchenden und Flüchtlingen in Deutschland. J Health Monit 2(1):24-47. https://doi.org/10. 17886/RKI-GBE-2017-005

8. BAfF-Bundesweite Arbeitsgemeinschaft Psychosozialer Zentren für Flüchtlinge und Folteropfer e. V. (2019) Versorgungsbericht zur psychosozialen Versorgung von Flüchtlingen und Folteropfern in Deutschland, 5. Aufl. BAfF, Berlin

9. United Nations, Economic and Social Council. Concluding observations on the sixth periodic report of Germany. 27 November 2018. E/C.12/DEU/CO/6. http://docstore. ohchr.org/SelfServices/FilesHandler.ashx?enc= 4sIQ6QSmIBEDzFEovLCuWx2r5QgrDoHhDa4Hdz LZSD2zbo\%2fzew8fG\%2f\%2fJWzgalqrl\%2fpQd KVEU\%2beWBy150Cs\%2f\%2bnkU3s6ayod026S tGVH8b0gDad0d4wZZ5\%2fvp6F1W5k\%2bvv

10. Razum O, Wenner J, Bozorgmehr K (2016) Wenn Zufall über den Zugang zur Gesundheitsversorgung bestimmt: Geflüchtete in Deutschland. Gesundheitswesen 78(11):711-714. https://doi. org/10.1055/s-0042-116231

11. Kühne A, Gilsdorf A (2016) Ausbrüche von Infektionskrankheiten in Gemeinschaftsunterkünften für Asylsuchende 2004-2014 in Deutschland. Bundesgesundheitsblatt Gesundheitsforschung Gesundheitsschutz 59(5):570-577. https://doi. org/10.1007/s00103-016-2332-9

12. Bozorgmehr K, Mohsenpour A, Saure D et al (2016) Systematische Übersicht und „Mapping" empirischerStudien des Gesundheitszustandsund der medizinischen Versorgung von Flüchtlingen und Asylsuchenden in Deutschland (1990-2014) Bundesgesundheitsblatt 59(5):599-620. https:// doi.org/10.1007/s00103-016-2336-5

13. BuffL, Röhrig B (2019) Zahnmedizinischer Behandlungsbedarf bei Asylbewerbern in RheinlandPfalz. Gesundheitswesen 81(08/09):749

14. Statistisches Bundesamt (2019) Sozialleistungen. Leistungen an Asylbewerber 2017, Erschienen am 23. August 2018, korrigiert am 09. Januar 2019. Fachserie 13 Reihe 7. Destatis, Wiesbaden

15. Forschungsdatenzentrum (FDZ) der Statistischen Ämter des Bundes und der Länder (2018) Empfänger von Asylbewerberregelleistungen (EVAS 22221)

16. Forschungsdatenzentrum (FDZ) der Statistischen Ämter des Bundes und der Länder (2018) Empfänger von besonderen Asylbewerberleistungen (EVAS 22231)

17. GENESIS-Onlinedatenbank des Statistischen Bundesamtes (2020) Asylbewerberleistungsstatistik "Statistik der Ausgaben und Einnahmen für Asylbewerberleistungen". https://www-genesis. destatis.de. Zugegriffen: 13. Jan. 2020

18. Robert Koch-Institut (RKI) (2015) Konzept zur Umsetzung frühzeitiger Impfungen bei Asylsuchenden nach Ankunft in Deutschland. Epidemiol Bull.https://doi.org/10.17886/EPIBULL-2015-011. 4

19. Walther L, Fuchs LM, Schupp J, von Scheve C (2020) Living conditions and the mental health and wellbeing of refugees: evidence from a large-scale German survey. JImmigr Minor Health. https://doi. org/10.1007/s10903-019-00968-5

20. Bozorgmehr K, Hintermeier M, Razum $O$ et al (2020) SARS-CoV-2 in Aufnahmeeinrichtungen und Gemeinschaftsunterkünften für Geflüchtete: Epidemiologische und normativ-rechtliche Aspek te. 2020, Bremen: Kompetenznetz Public Health COVID-19. Version1.0, 29.05.2020. https://pub. uni-bielefeld.de/download/2943665/2943668/ FactSheet_PHNetwork-Covid19_Aufnahmeein richtungen_v1_inkl_ANNEX.pdf 